\title{
Numerical Simulation of Non-Linear Schrödinger Equations in Arbitrary Domain by the Localized Method of Approximate Particular Solution
}

\author{
Yongxing Hong ${ }^{1}$, Jun $\mathrm{Lu}^{2,3, *}$, Ji Lin ${ }^{1}$ and Wen Chen ${ }^{1}$ \\ 1 State Key Laboratory of Hydrology-Water Resources and Hydraulic Engineering, \\ International Center for Simulation Software in Engineering and Sciences, College of \\ Mechanics and Materials, Hohai University, Nanjing 211100, China \\ 2 Nanjing Hydraulic Research Institute, Hujuguan 34 Road, Nanjing 210024, China \\ 3 State Key Laboratory of Hydrology-Water Resources and Hydraulic Engineering, \\ Nanjing 210098, China
}

Received 23 January 2018; Accepted (in revised version) 21 September 2018

\begin{abstract}
The aim of this paper is to propose a fast meshless numerical scheme for the simulation of non-linear Schrödinger equations. In the proposed scheme, the implicitEuler scheme is used for the temporal discretization and the localized method of approximate particular solution (LMAPS) is utilized for the spatial discretization. The multiple-scale technique is introduced to obtain the shape parameters of the multiquadric radial basis function for 2D problems and the Gaussian radial basis function for 3D problems. Six numerical examples are carried out to verify the accuracy and efficiency of the proposed scheme. Compared with well-known techniques, numerical results illustrate that the proposed scheme is of merits being easy-to-program, high accuracy, and rapid convergence even for long-term problems. These results also indicate that the proposed scheme has great potential in large scale problems and real-world applications.
\end{abstract}

AMS subject classifications: 00A35

Key words: Schrödinger equation, Localized method of approximate particular solution, Shape parameters, Multiple-scale technique.

\section{Introduction}

In this paper, we consider the following general form of the non-linear Schrödinger equation:

$$
i \frac{\partial u(x, t)}{\partial t}+a \Delta u+w(x, t) u(x, t)+v(x, t)|u(x, t)|^{m} u(x, t)=0,
$$

\footnotetext{
*Corresponding author.

Email: lujunhhu1981@126.com (J. Lu)
} 
under the following initial condition

$$
u(x, t)=u_{0}(x, t), \quad x \in \Omega, \quad t=0,
$$

and boundary conditions

$$
\begin{gathered}
\frac{\partial u(x, t)}{\partial n}=f(x, t), \quad x \in \Gamma_{\mathrm{N}}, \quad t \in[0, T], \\
u(x, t)=g(x, t), \quad x \in \Gamma_{d}, \quad t \in[0, T],
\end{gathered}
$$

where $i=\sqrt{-1}, T$ is the final time, $t$ denotes the time history, $u(x, t)$ is an unknown wave function to be determined, $\Delta$ is the Laplace operator, $\omega(x, t)$ and $v(x, t)$ are known functions and $m$ is a positive real constant, $u_{0}, g$, and $f$ are given functions, $\Omega$ denotes the interest domain, $\Gamma_{\mathrm{N}}$ and $\Gamma_{d}$ represent boundaries under the Neumann and Dirichlet boundary conditions respectively.

The Schrödinger equations describe various types of physical phenomena in science and engineering. Korepin and Zhang utilized Schrödinger equations to depict wavecorpuscle duality of microscopic particles in quantum mechanics [1,2]. Onorato and Osborne analyzed the water wave propagation using the Schrödinger equations $[3,4]$, which can also be used on ocean environmental description and the design of ships and shore structures $[5,6]$. The Schrödinger equations have also been used to describe the standing waves [7], electromagnetic fields [8], electro-optic wave propagation [9], as well as the structures of biological materials $[10,11]$.

In recent years, various numerical algorithms have been proposed to solve the Schrödinger equations. It is known to all that the tradition element-based methods are very powerful and effective tools for solving problems in science and engineering such as the finite difference method, the finite element method, and the boundary element method $[12,13]$. Anastassi has proposed the finite difference method in solving the Schrödinger equations and related oscillatory problems [14]. Zhu employed the finite element method for the time-space-fractional Schrödinger equation [15]. To avoid the mesh of the solution domain, the boundary element method reduces the dimension of the problem by one [16]. Zhao has improved the accuracy in solving the fractional Schrödinger equation by the alternating direction implicit scheme [17]. Bhrawy introduced an effective spectral scheme to solve the multi-dimensional space-time variable-order fractional Schrödinger equations [18]. Zhang has proposed the improved complex variable moving least-squares Ritz method and the improved complex variable element-free Galerkin method for Schrödinger equations [19,20].

In the last two decades, the radial basis functions (RBFs) based methods have attracted great attention and enjoyed considerable success in solving partial difference equations (PDEs) [21-24]. These approaches can be easily extended to solve high dimensional problems due to its spatial dimension independence, and applied to solve high order differential equations due to the smooth characteristic of RBFs [25-28]. It should be noted that there are some drawbacks for the radial basis functions based methods. For some RBFs, it is difficult to obtain the optimal shape parameters, such as the 
multiquadric radial basis functions (MQ-RBFs) [29] and the Gaussian radial basis functions [30,31], which is crucial to the accuracy of the numerical algorithms. What's worse, the coefficient matrix can be highly ill-conditioned for large scale problems [32]. In order to overcome these drawbacks, researchers come up with the localized approaches including the compactly supported RBFs, the localized RBFs collocation method, the local radial basis functions based differential quadrature collocation method [33,34], and the localized method of approximate particular solutions (LMAPS) [35]. However, the determination of shape parameters in RBFs remains an open issue [36] even for the localized scheme.

In this paper, we propose the localized method of approximate particular solution (LMAPS) to solve the 2D and 3D non-linear time-dependent Schrödinger equations. In the proposed scheme, the multiple-scale technique (MST) is introduced for the determination of the shape parameters in MQ-RBF to solve 2D problems. The MST is first proposed to determine the shape parameters of MQ-RBF in the Kansa's method [37]. The MST requires the norm of all the rows or columns of an equilibrated matrix to be the same. Thus, the shape parameters can be easily defined especially for the LMAPS due to the characteristics of the localized scheme. Furthermore, we extended the MST to $3 \mathrm{D}$ problems based on Gaussian radial basis functions. In the LMAPS, the k-d tree algorithm [38] is applied for searching the neighboring points of each local center. Recently, the LMAPS has been successfully applied for the simulation of heat conduction, the molecular dynamics, the wave propagation, and the flow fields [39-42].

The layout of the rest of this paper is as follows: In Section 2, we present a localized meshless scheme and its application for 2D and 3D non-linear Schrödinger equations. In Section 3, numerical results for six examples are obtained and compared with analytical results to test the accuracy, stability, convergence and efficiency of the proposed scheme. Concluding remarks are given in Section 4.

\section{A localized meshless scheme for Schrödinger equations}

In this section, we employ the time stepping method to deal with the time derivative in Eq. (1.1). Then, we introduce the LMAPS which is used for the corresponding spatial discretization.

\subsection{Time discretization of Schrödinger equation}

Firstly, the time interval $[0, T]$ is divided into $S$ equally sub-intervals, with the time step size $d t=T / S$ and $t^{n}=n \cdot d t, n=0,1, \cdots, S$, where $n$ is positive real number. Then the implicit-Euler scheme is presented here to discretize Eq. (1.1) as follows:

$$
\mathrm{i} \frac{u^{n+1}-u^{n}}{d t}+a \Delta u^{n+1}+\omega^{n+1} u^{n+1}+v^{n+1}\left|u^{n+1}\right|^{m} u^{n+1}=0
$$


where $u^{n+1}$ is the approximation of $u\left(x, t^{n+1}\right), \omega^{n+1}$ denotes $\omega\left(x, t^{n+1}\right)$, and $v^{n+1}$ denotes $v\left(\boldsymbol{x}, t^{n+1}\right)$. Rearranging Eq. (2.1) by moving the parts including $u^{n}$ to the right-hand-side of the equation yields

$$
-\mathbf{i} u^{n+1}-a d t \Delta u^{n+1}-d t \cdot \omega\left(x, t^{n+1}\right) u^{n+1}-d t \cdot v^{n+1}\left|u^{n+1}\right|^{m} u^{n+1}=-\mathbf{i} u^{n} .
$$

\subsection{The localized method of approximate particular solutions}

In the LMAPS, the interest domain is divided into several sub-domains, and the localized low-rank matrix is formed based on the local nodes in each sub-domain. Then a sparse system of equations is built up by reformulating the localized form into globalized form.

For convenience, we first introduce the global approximation strategy and denote $\left\{x_{j}\right\}_{j=1}^{N}$ as the interpolation points of the interest domain $\Omega$. The value of $u(x)$ can be approximated by a linear combination of $N$ function values in the global form:

$$
u(x) \approx \sum_{j=1}^{N} \alpha_{j} \Phi\left(\left\|x-x_{j}\right\|_{2}\right)
$$

also we have

$$
\Delta u(\boldsymbol{x}) \approx \Delta \hat{u}(\boldsymbol{x})=\sum_{j=1}^{N} \alpha_{j} \Delta \Phi\left(\left\|\boldsymbol{x}-\boldsymbol{x}_{j}\right\|_{2}\right),
$$

where

$$
\Delta \Phi\left(\left\|x-x_{j}\right\|_{2}\right)=\phi\left(\left\|x-x_{j}\right\|_{2}\right),
$$

where $\phi\left(\left\|x-x_{j}\right\|_{2}\right)$ are RBFs. In this paper, the MQ-RBFs are selected, since they have been found to be able to provide very accurate approximations in the most of applications and have been widely used by researchers $[43,44]$. The MQ-RBF is defined as follows:

$$
\phi_{i j}=\sqrt{\left\|x_{i}-x_{j}\right\|_{2}^{2}+c^{2}}
$$

and the normalized Gaussian radial basis function is written as

$$
\breve{\phi}_{i j}=\exp \left(-c\left\|x_{i}-x_{j}\right\|_{2}^{2}\right)
$$

where $c$ is the shape parameter.

For 2D problems, by direct integration from Eq. (2.6) with the notation $\left\|x-x_{j}\right\|_{2}=r$, we have

$$
\Phi(r)=\left(4 c^{2}+r^{2}\right) \phi(r) / 9-c^{3} \log (c+\phi(r)) / 3
$$

For $3 \mathrm{D}$ problems, by direct integration from Eq. (2.7), we have

$$
\Phi(r)= \begin{cases}\frac{-\sqrt{\pi}}{4 c^{3 / 2} r} \operatorname{erf}(\sqrt{c} r), & r \neq 0, \\ \frac{-1}{2 c}, & r=0\end{cases}
$$


where the erf is a special function known as error function for each of $p$ written as follows

$$
\operatorname{erf}(p)=\frac{2}{\sqrt{\pi}} \int_{0}^{p} e^{-q^{2}} d q
$$

Then Eq. (2.3) can be rewritten in the following matrix form:

$$
\hat{u}=A_{\Phi} \alpha,
$$

where, using the notation $\Phi\left(\left\|x_{i}-x_{j}\right\|_{2}\right)=\Phi_{i j}$ and $\hat{u}\left(x_{i}\right)=\hat{u}_{i}, A_{\Phi}=\left[\Phi_{i j}\right]_{n \times n}, \alpha=$ $\left[\alpha_{1}, \alpha_{2}, \cdots, \alpha_{N}\right]^{T}$, and $\hat{\boldsymbol{u}}=\left[\hat{u}_{1}, \hat{u}_{2}, \cdots, \hat{u}_{N}\right]^{T}$. The coefficient vector $\boldsymbol{\alpha}$ can be obtained from Eq. (2.11) as follows:

$$
\alpha=A_{\Phi}^{-1} \hat{u} .
$$

So the derivative of $u(x)$ can be approximated by $\mathcal{D} \hat{u}(x)=\sum_{j=1}^{N} \alpha_{j} \mathcal{D} \Phi\left(\left\|x-x_{j}\right\|_{2}\right)$, then

$$
\mathcal{D} \hat{u}(x)=A_{\mathcal{D}} \alpha, \quad x \in \Omega,
$$

where $\mathcal{D}$ is the derivative operator, $A_{\mathcal{D}}=\left[\mathcal{D} \Phi\left(\left\|x-x_{1}\right\|_{2}\right), \mathcal{D} \Phi\left(\left\|x-x_{2}\right\|_{2}\right), \cdots, \mathcal{D} \Phi(\| x-\right.$ $\left.x_{N} \|_{2}\right)$ ]. Substituting Eq. (2.12) into Eq. (2.13), we have

$$
\mathcal{D} \hat{u}(x)=A_{\mathcal{D}} A_{\Phi}^{-1} \hat{u} .
$$

Then, the solution domain is divided into several sub-domains by setting a center point in each sub-domain and selecting several points near the center point to support the subdomain. By applying the global MQ-RBF approximation strategy on each sub-domain, the low-rank matrix is formed as follows:

$$
\boldsymbol{A}_{i}=\left[\Phi_{k j}\right]_{n s \times n s}, \quad \boldsymbol{x}_{k}, \boldsymbol{x}_{j} \in \boldsymbol{\Omega}_{i},
$$

where $n s$ is the number of the support points in each sub-domain $\boldsymbol{\Omega}_{i}, \boldsymbol{x}_{i}$ is the selected center point, $k$ and $j$ are the serial numbers of the points close to the center point $x_{i}$. Using the local Multiquadric approximation strategy and performing Eq. (2.15) into Eq. (2.14) with the notation $[\boldsymbol{A}]^{-1}=\left[\bar{\Phi}_{k j}\right]_{n s \times n s}$ and $\mathcal{D} \Phi\left(\left\|\boldsymbol{x}_{i}-\boldsymbol{x}_{j}\right\|_{2}\right)=\mathcal{D} \Phi_{i j}$, the approximation of $\mathcal{D} u\left(\boldsymbol{x}_{i}\right)$ at the center point $x_{i}$ can be written as a linear combination of $n s$ MQ-RBFs in the following form

$$
\mathcal{D} \hat{u}_{i}=S_{i j} \cdot \hat{u}_{j},
$$

where $\mathcal{D} \hat{u}_{i}$ and $\mathcal{D} \hat{u}_{j}$ denote $\mathcal{D} \hat{u}\left(x_{i}\right)$ and $\mathcal{D} \hat{u}\left(x_{j}\right), \boldsymbol{x}_{j} \in \boldsymbol{\Omega}_{i}$, and $S_{i j}=\mathcal{D} \Phi_{i k} \cdot \bar{\Phi}_{k j}$. By setting $S_{i j}=0$, where $x_{j} \notin \boldsymbol{\Omega}_{i}$, we can reformulate the localized formulation into globalized form as follows

$$
\mathcal{D} \hat{u}_{i}=\sum_{j=1}^{N} S_{i j} \cdot \hat{u}_{j},
$$

where

$$
S_{i j}= \begin{cases}\mathcal{D} \Phi_{i k} \cdot \bar{\Phi}_{k j}, & x_{j} \in \boldsymbol{\Omega}_{i}, \\ 0, & x_{j} \notin \boldsymbol{\Omega}_{i},\end{cases}
$$

and most of the elements in $\left\{S_{i j}\right\}_{j=1}^{N}$ are zero. Thus, a sparse system of equations can be constructed. 


\subsection{Formulation of the LMAPS for Schrödinger equation}

Substituting the localized form Eq. (2.17) into Eq. (2.2) for $x_{i} \in \Omega$, we have

$$
-i \hat{u}_{i}^{n+1}-a d t \sum_{j=1}^{N} S_{i j} \hat{u}_{j}^{n+1}-d t \cdot \omega_{i}^{n+1} \hat{u}_{i}^{n+1}-d t \cdot v_{i}^{n+1}\left|\hat{u}_{i}^{n+1}\right|^{m} \hat{u}_{i}^{n+1}=-i \hat{u}_{i}^{n}, \quad 1 \leq i \leq n i,
$$

where

$$
S_{i j}= \begin{cases}\Delta \Phi_{i k} \cdot \bar{\Phi}_{k j}, & x_{j} \in \boldsymbol{\Omega}_{i}, \\ 0, & x_{j} \notin \boldsymbol{\Omega}_{i},\end{cases}
$$

$\left|\hat{u}_{i}^{n+1}\right|^{m}$ can be replaced by $\left|\hat{u}_{i}^{n}\right|^{m}$ in the computation, which is the latest approximation of $\left|\hat{u}_{i}^{n+1}\right|^{m}$, and $n i$ is the number of the interpolation nodes inside interest domain $\Omega$. Then we add that $\delta_{i j}$ has the Kronecker delta function property, that is

$$
\delta_{i j}= \begin{cases}1, & i=j, \\ 0, & i \neq j .\end{cases}
$$

Then, the Eq. (2.19) can be written as

$$
\left(-i-d t \cdot \omega_{i}^{n+1}-d t \cdot v_{i}^{n+1}\left|u_{i}^{n}\right|^{m}\right) \boldsymbol{E}_{i} \hat{\boldsymbol{u}}^{n+1}-a d t s_{i} \hat{\boldsymbol{u}}^{n+1}=-i \boldsymbol{E}_{i} \hat{\boldsymbol{u}}^{n}, \quad 1 \leq i \leq n i,
$$

where $\boldsymbol{E}_{i}=\left[\delta_{i 1}, \delta_{i 2}, \cdots, \delta_{i N}\right], \boldsymbol{S}_{i}=\left[S_{i 1}, S_{i 2}, \cdots, S_{i N}\right]$, and $\hat{\boldsymbol{u}}^{n+1}=\left[\hat{u}_{1}^{n+1}, \hat{u}_{2}^{n+1}, \cdots \hat{u}_{N}^{n+1}\right]^{T}$. By using the notation $\left(-i-d t \cdot \omega_{i}^{n+1}-d t \cdot v_{i}^{n+1}\left|u_{i}^{n}\right|^{m}\right) \boldsymbol{E}_{i}-a d t \boldsymbol{S}_{i}=\boldsymbol{L}_{i}$, Eq. (2.22) can be written as

$$
\boldsymbol{L}_{i} \cdot \hat{\boldsymbol{u}}^{n+1}=-\mathrm{i} \boldsymbol{E}_{i} \hat{\boldsymbol{u}}^{n}, \quad 1 \leq i \leq n i .
$$

To consider the Neumann boundary condition of Eq. (1.3a) for $x_{i} \in \Gamma_{N}$, we have

$$
\boldsymbol{S}_{i} \cdot \hat{\boldsymbol{u}}^{n+1}=f_{i}^{n+1}, \quad n i+1 \leq i \leq n i+n b_{1},
$$

where $f_{i}^{n+1}=f\left(x_{i}, t^{n+1}\right)$,

$$
S_{i j}= \begin{cases}\frac{\partial \Phi_{i k}}{\partial \boldsymbol{n}_{i}} \cdot \bar{\Phi}_{k j}, & \boldsymbol{x}_{j} \in \boldsymbol{\Omega}_{i}, \\ 0, & \boldsymbol{x}_{j} \notin \mathbf{\Omega}_{i},\end{cases}
$$

and to consider the Dirichlet boundary condition of Eq. (1.3b) for $x_{i} \in \Gamma_{D}$, we have

$$
\boldsymbol{E}_{i} \hat{\boldsymbol{u}}^{n+1}=g_{i}^{n+1}, \quad n i+n b_{1}+1 \leq i \leq n i+n b_{1}+n b_{2},
$$

where $\boldsymbol{n}_{i}$ denotes the unit outward normal vector to the boundary node $\boldsymbol{x}_{i}, g_{i}^{n+1}=$ $g\left(\boldsymbol{x}_{i}, t^{n+1}\right), n b_{1} / n b_{2}$ is the number of the interpolation nodes on the Neumann/Dirichlet boundary part.

Then, combining Eqs. (2.23)-(2.26), we can rewritten them in the following matrix form:

$$
\boldsymbol{B} \cdot \hat{\boldsymbol{u}}^{n+1}=\tilde{\boldsymbol{u}}^{n},
$$


where

$$
\begin{aligned}
& \boldsymbol{B}_{i}=\left\{\begin{array}{l}
\boldsymbol{L}_{i}, \quad 1 \leq i \leq n i, \\
\boldsymbol{S}_{i,} n i+1 \leq i \leq n i+n b_{1}, \\
\boldsymbol{E}_{i,} n i+n b_{1}+1 \leq i \leq N,
\end{array}\right. \\
& \tilde{\boldsymbol{u}}_{i}^{n}= \begin{cases}-i u_{i}, & 1 \leq i \leq n i, \\
f_{i}^{n+1}, & n i+1 \leq i \leq n i+n b_{1}, \\
g_{i}^{n+1}, & n i+n b_{1}+1 \leq i \leq N .\end{cases}
\end{aligned}
$$

Thus, $\hat{\boldsymbol{u}}^{n+1}$ can be solved as follows:

$$
\hat{\boldsymbol{u}}^{n+1}=\boldsymbol{B}^{-1} \cdot \tilde{\boldsymbol{u}}^{n} .
$$

It can be seen that most of the elements in $\boldsymbol{B}$ are equal to zero, so it can be represented by a sparse matrix and solved efficiently.

\subsection{Determination of the shape parameters}

As the MST described in [37], considering the MQ-RBF, and according to Eq. (2.26), we can acquire that the norm of every sparse system should be equal to 1 , thus we have

$$
\sum_{j=1}^{n s}\left\|\boldsymbol{x}_{i}-\boldsymbol{x}_{j}\right\|_{2}^{2}+n s \cdot c_{i}^{2}=1^{2}, \quad \boldsymbol{x}_{j} \in \mathbf{\Omega}_{i}
$$

Then, the $c_{i}$ can be determined as

$$
c_{i}=\sqrt{\frac{1}{n s}\left(1-\sum_{j=1}^{n s}\left\|x_{i}-x_{j}\right\|_{2}^{2}\right)} .
$$

As for the Gaussian function in Eq. (2.7), when the $\left\|x_{i}-x_{j}\right\|_{2}^{2}$ is close to be zero, the value of the Gaussian function turns close to 1. Let us multiply both sides of Eq. (2.26) by $\sqrt{n s}$ written as

$$
\sqrt{n s} \boldsymbol{E}_{i} \hat{\boldsymbol{u}}^{n+1}=\sqrt{n s} g_{i}^{n+1}, \quad n i+n b_{1}+1 \leq i \leq n i+n b_{1}+n b_{2} .
$$

Thus, the norm of every sparse system should be equal to $\sqrt{n s}$. We have

$$
\sum_{j=1}^{n s} e^{-2 c_{i}\left\|x_{i}-x_{j}\right\|_{2}^{2}}=n s .
$$

Here, using the notation of $e^{-1 / n s} \approx 1$ and considering the average value of the summation of $\left\|x_{i}-x_{j}\right\|_{2}^{2}$, we have

$$
n s \cdot e^{-2 c_{i} \frac{1}{n s} \sum_{j=1}^{n s}\left\|x_{i}-x_{j}\right\|_{2}^{2}} \approx n s \cdot e^{-1 / n s},
$$


and taking the logarithm on both sides, it comes

$$
-2 c_{i} \frac{1}{n s} \sum_{j=1}^{n s}\left\|\boldsymbol{x}_{i}-\boldsymbol{x}_{j}\right\|_{2}^{2}=-\frac{1}{n s} .
$$

Thus the $c_{i}$ can be achieved as

$$
c_{i}=\frac{1}{2 \sum_{j=1}^{n s}\left\|x_{i}-x_{j}\right\|_{2}^{2}}
$$

\section{Numerical results}

To illustrate the validity, convergence, stability and efficiency of the proposed scheme, six examples of Eqs. (1.1)-(1.3b) with $m=2$ are considered. The numerical results are compared with the analytical solutions. For Examples 3.1-3.4, the analytical solutions can be found in $[45,46]$. The root mean square error $(R M S)$ is reported to validate the accuracy of numerical results which is defined as follows:

$$
R M S=\sqrt{\frac{1}{n_{t}} \sum_{i=1}^{n_{t}}\left(\hat{u}_{i}-u_{i}\right)^{2}},
$$

where $n_{t}$ denotes the total number of test points.

Example 3.1. In the first example, the accuracy of the proposed scheme is tested on a linear Schrödinger equation with potential functions

$$
\omega\left(x_{1}, x_{2}, t\right)=-\frac{4 x_{1}^{2}+4 x_{2}^{2}-4 x_{1}-4 x_{2}+\beta^{2}-4 \beta+2}{\beta^{2}}
$$

with $v=0$ and $a=1$. The computational domain is $\left[x_{1}, x_{2}\right] \in[0,1] \times[0,1]$ with uniformly distributed nodes where $N=32^{2}$. The analytical solution is

$$
u\left(x_{1}, x_{2}, t\right)=\exp \left[-\frac{\left(x_{1}-0.5\right)^{2}+\left(x_{2}-0.5\right)^{2}}{\beta}-i t\right] .
$$

In this example, $\beta=0.05, n s=13$, and $d t=0.005(\mathrm{~s})$. Fig. 1 presents the comparison of the analytical solutions and numerical solutions at $T=1(\mathrm{~s})$, where $\operatorname{real}(u) / \operatorname{imag}(u)$ denotes the real/imaginary part of analytical solutions and real $(\hat{u}) / \operatorname{imag}(\hat{u})$ denotes the real/imaginary part of numerical solutions. From this figure, it can be seen that the numerical solutions are consistent with the analytical solutions. Then, Fig. 2 shows the absolute errors at $T=1(\mathrm{~s})$. The maximum errors of both parts are around $10^{-3}$. Fig. 3 presents the comparison of the analytical solutions and numerical solutions at $T=2(\mathrm{~s})$ and Fig. 4 shows more details about their difference. From the two figures, it can be found that the numerical solutions are very close to the analytical solutions with errors less than $10^{-2}$. All these results demonstrate the accuracy of the proposed scheme. 

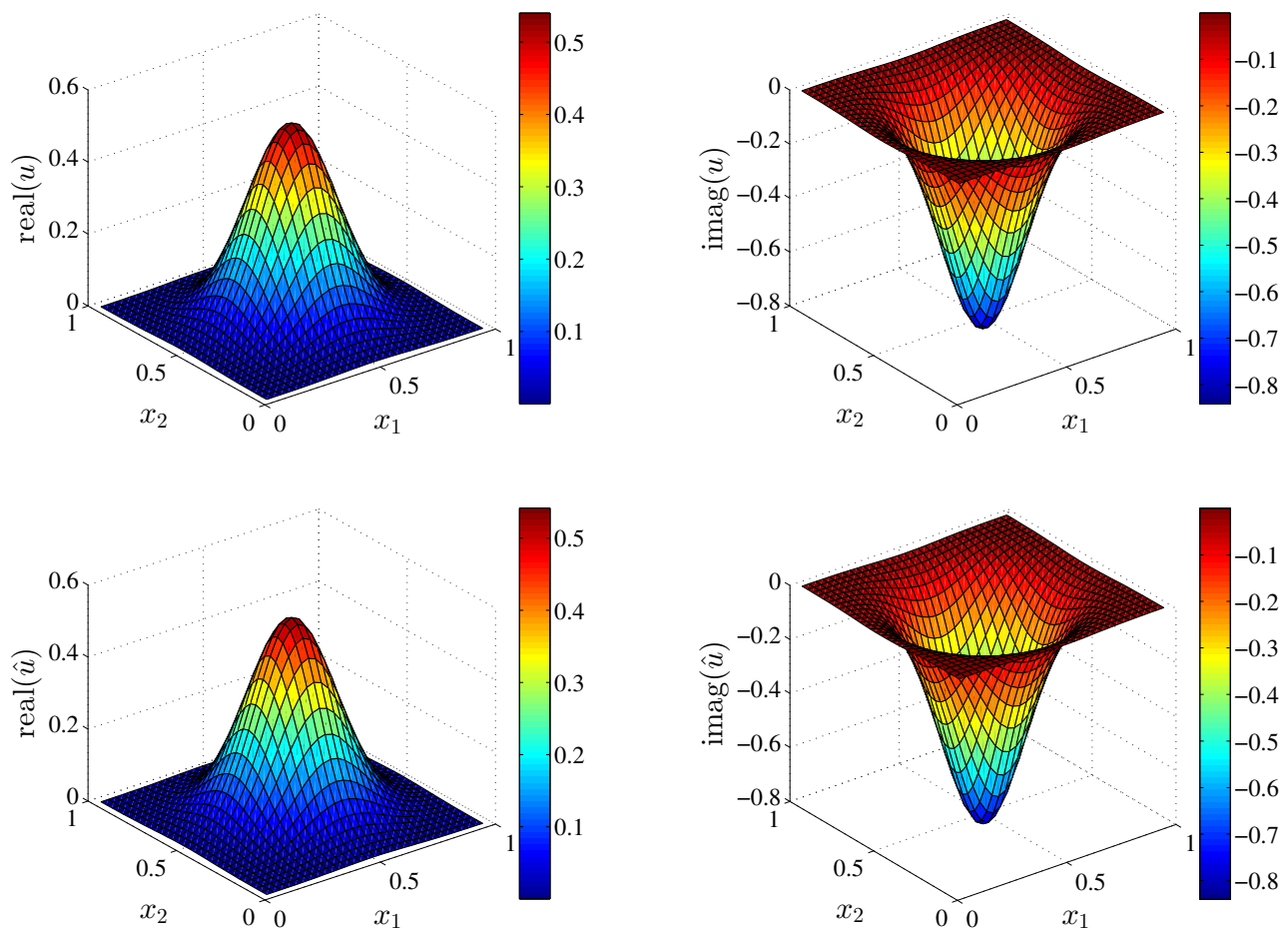

Figure 1: The comparison of the analytical solutions and numerical solutions at $T=1(\mathrm{~s})$ for Example 3.1.
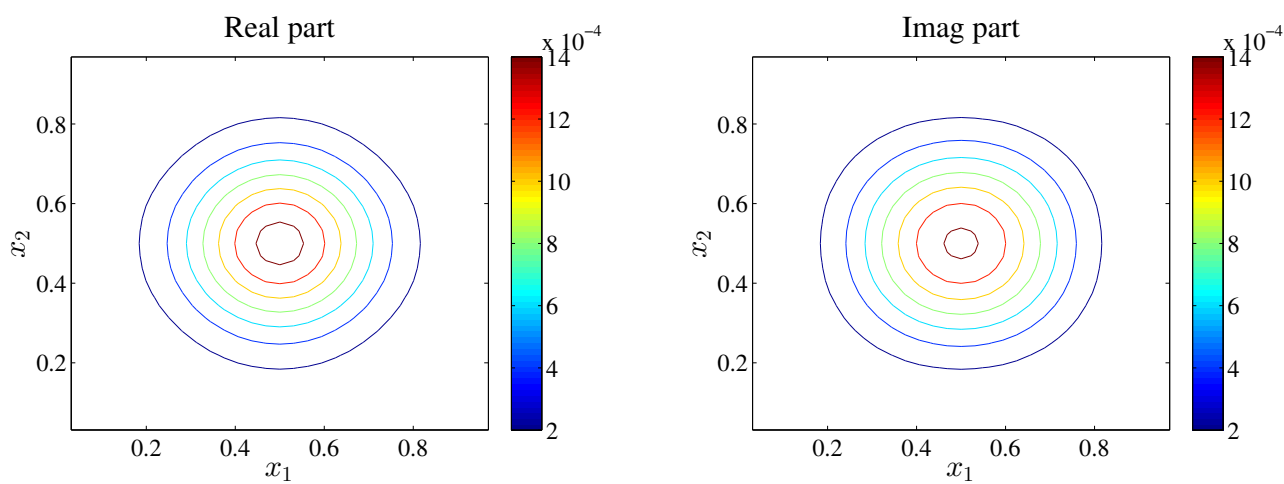

Figure 2: The absolute errors in the interest domain at $T=1(\mathrm{~s})$ for Example 3.1.

Example 3.2. To further show the convergency of the proposed scheme, the second example is designed with potential functions $v=q$ and $\omega\left(x_{1}, x_{2}, t\right)=0$, where $a=1$. The results obtained by the proposed method are compared with those by using the "optimal" shape parameter $\left(c_{\text {optimal }}\right)$ and the leave-one-out cross validation (LOOCV) algorithm. The ana- 

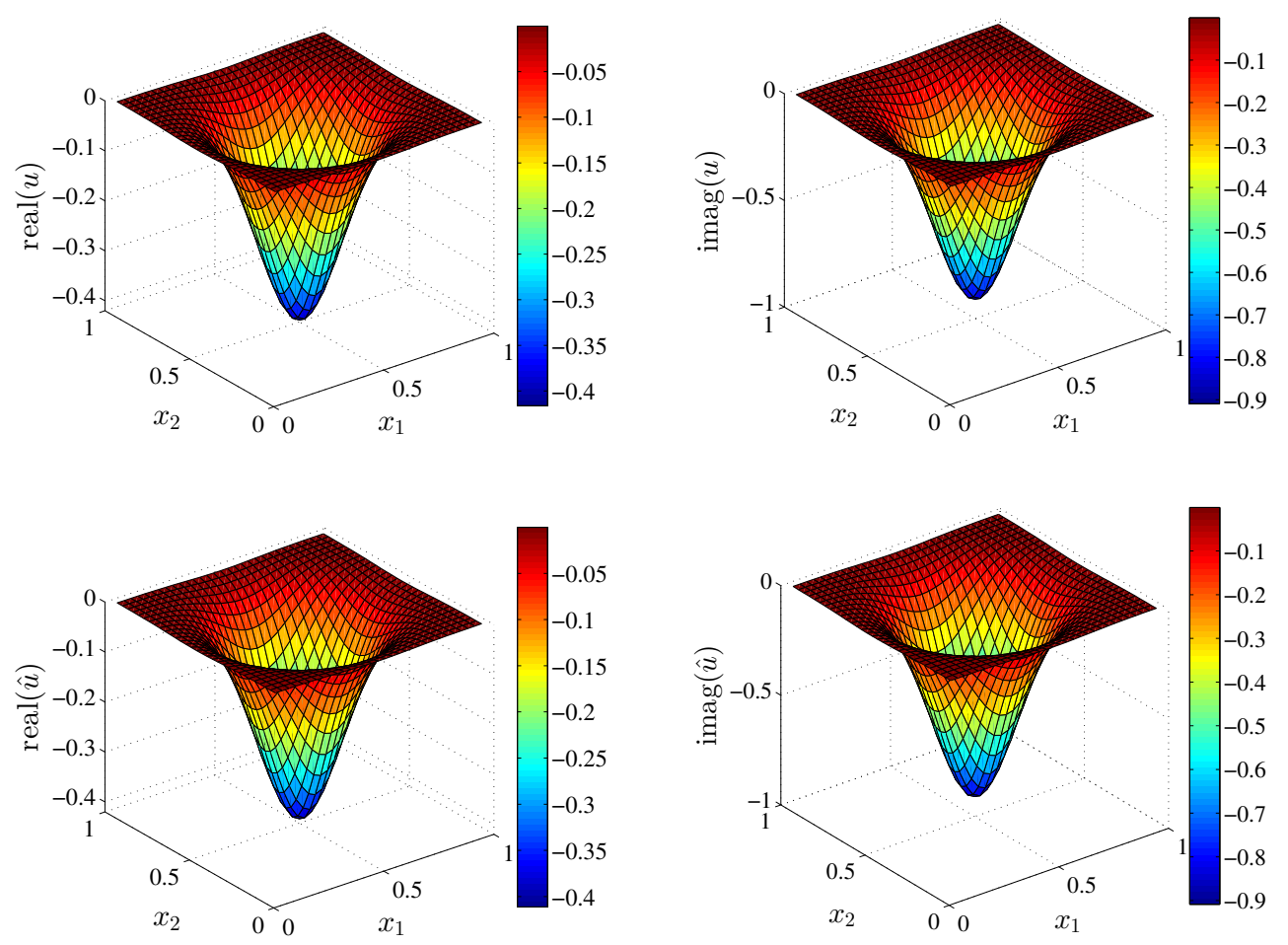

Figure 3: The comparison of the analytical solutions and numerical solutions at $T=2(\mathrm{~s})$ for Example 3.1.
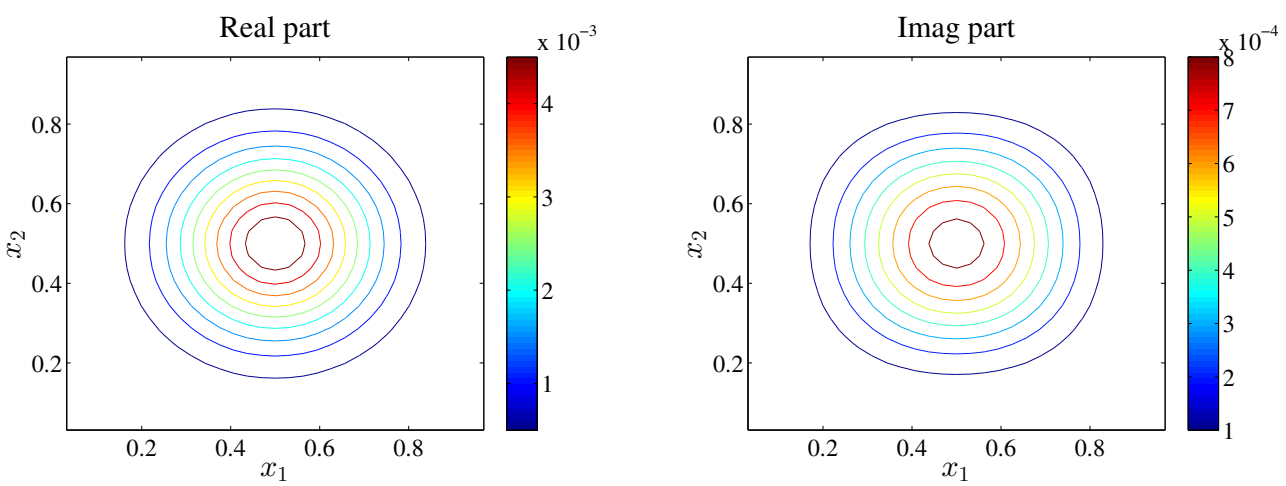

Figure 4: The absolute errors in the interest domain at $T=2(\mathrm{~s})$ for Example 3.1.

lytical solution of this problem can be written as

$$
u\left(x_{1}, x_{2}, t\right)=A \exp \left[i\left(k_{1} x_{1}+k_{2} x_{2}-\beta t\right)\right],
$$

where $A, k_{1}, k_{2}$, and $q$ are constants and $\beta=k_{1}^{2}+k_{2}^{2}-q|A|^{2}$. The numerical experiment is carried out on the bone region with irregular distributed nodes as shown in Fig. 5, 


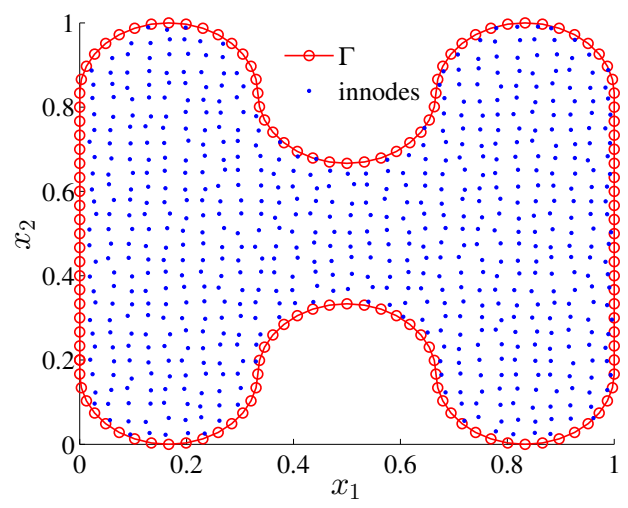

Figure 5: The interest domain and the distribution of collocation nodes for Example 3.2.
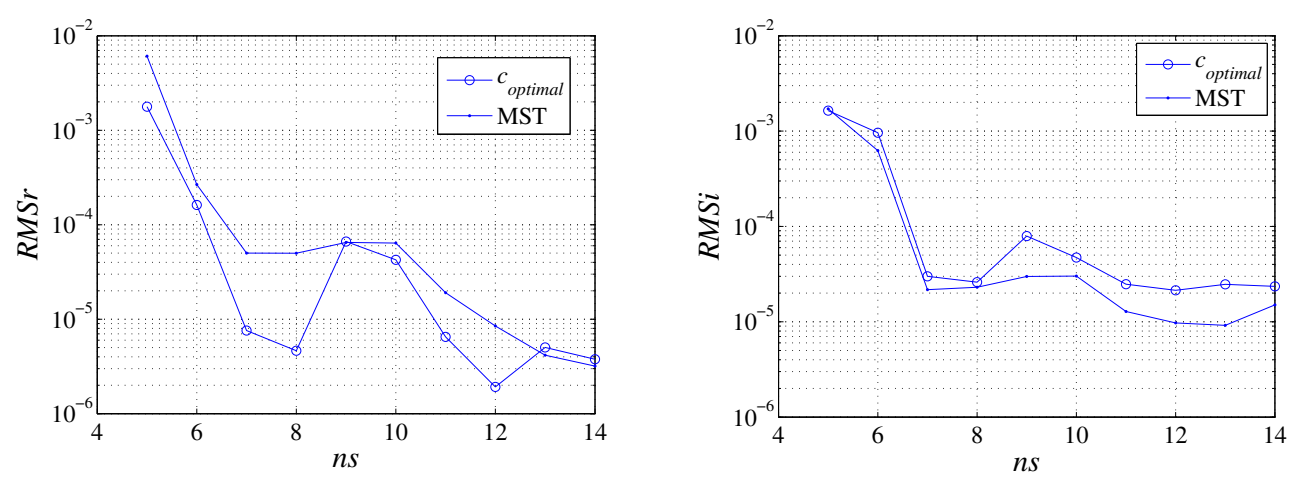

Figure 6: The comparison of the RMS versus $n s$ obtained by using $c_{\text {optimal }}$ and MST at $T=1(\mathrm{~s})$ with $d t=0.001(\mathrm{~s})$, where the $c_{\text {optimal }}$ versus $n s$ is as shown in Table 1 for Example 3.2.

where $\Gamma$ denotes the closed curve of the interest domain, the total number of collocation nodes is $N=767$ containing 631 interior nodes and 136 boundary nodes. In this example, $A=k_{1}=k_{2}=q=1$, we have $\beta=1$. In Fig. 6, the comparison of the $R M S$ versus $n s$ obtained by using the $c_{\text {optimal }}$ and the proposed MST is shown, where $R M S r / R M S i$ denotes the real/imaginary part of the RMS and the $c_{\text {optimal }}$ versus $n s$ is as shown in Table 1 . It can be seen that the MST can achieve accurate results as the $c_{\text {optimal }}$ with exponential convergence. When $n s$ is equal to 13 or 14 , they achieve the similar RMS with RMSr around $10^{-6}$ and $R M S i$ around $10^{-5}$. It means that the proposed MST is good enough for automatically determining the shape parameters. Fig. 7 makes the comparison of their elapsed time. From this figure, it can be seen that the MST needs just 0.5 second compared with $c_{\text {optimal }}$. Fig. 8 presents the comparison of the RMS versus $N$ obtained by using the LOOCV and the MST at $T=1$ (s) with $d t=0.001(\mathrm{~s})$. As shown in this figure, the $R M S r$ and RMSi decrease as the total number of collocation nodes $N$ increases by both of the LOOCV and the MST. Meanwhile, the results obtained by the MST are a bit more 


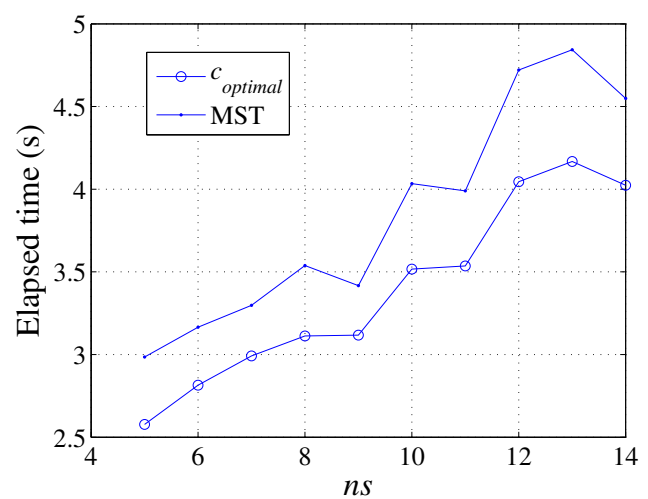

Figure 7: The comparison of the elapsed times versus $n s$ obtained by using $c_{\text {optimal }}$ and MST at $T=1$ (s) with $d t=0.001(\mathrm{~s})$ for Example 3.2.
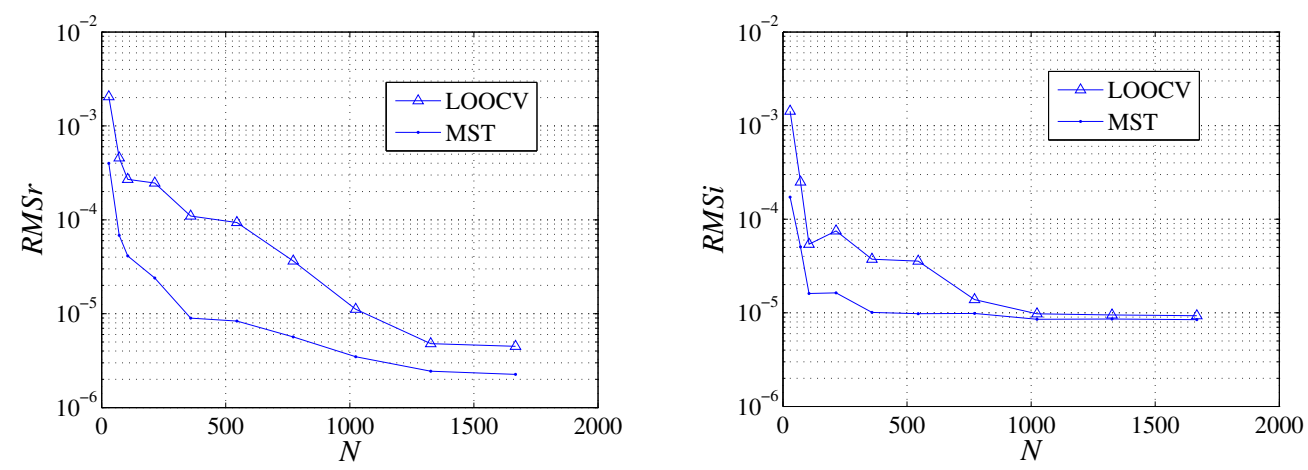

Figure 8: The comparison of the RMS versus $N$ obtained by using LOOCV and MST at $T=1(\mathrm{~s})$ with $d t=0.001(\mathrm{~s})$ for Example 3.2.

accurate than those obtained by the LOOCV. Fig. 9 shows their elapsed time respectively. From this figure, we can find that the elapsed time of the MST or the LOOCV increases as the total number of collocation nodes increase and the MST is faster than the LOOCV. Moreover, Fig. 10 displays the $R M S$ versus the time step $d t$ obtained at $T=1(\mathrm{~s})$. It can be found that the smaller $d t$ is used, the higher accuracy can be achieved. All these results demonstrate the convergency and efficiency of the proposed scheme.

Example 3.3. We consider a nonlinear Schrödinger equation with potential functions

Table 1: The optimal parameter $c$ versus $n s$ for Fig. 6 , where $d t=0.001$ and $d_{1}$ denotes the maximum distance to center node in sub-domain.

\begin{tabular}{||ccccccccccc||}
\hline$n s$ & 5 & 6 & 7 & 8 & 9 & 10 & 11 & 12 & 13 & 14 \\
\hline$c\left(\times d_{1}\right)$ & 500 & 200 & 50 & 50 & 19 & 19 & 20 & 20 & 14 & 14 \\
\hline
\end{tabular}




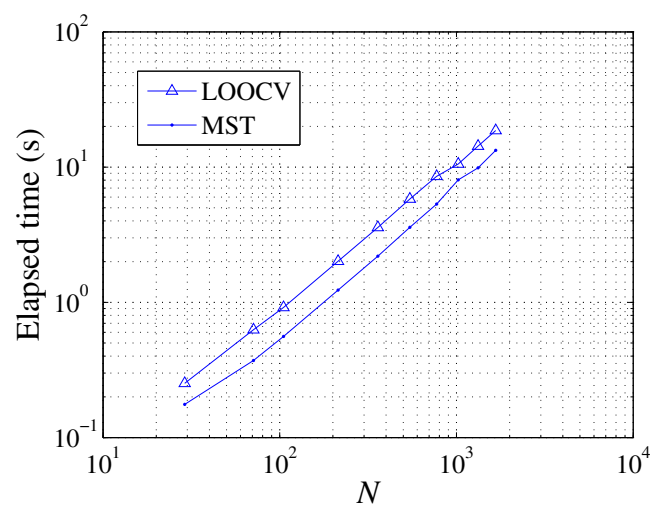

Figure 9: The comparison of the elapsed time versus $N$ obtained by LOOCV and MST at $T=1(\mathrm{~s})$ with $d t=0.001(\mathrm{~s})$ for Example 3.2.

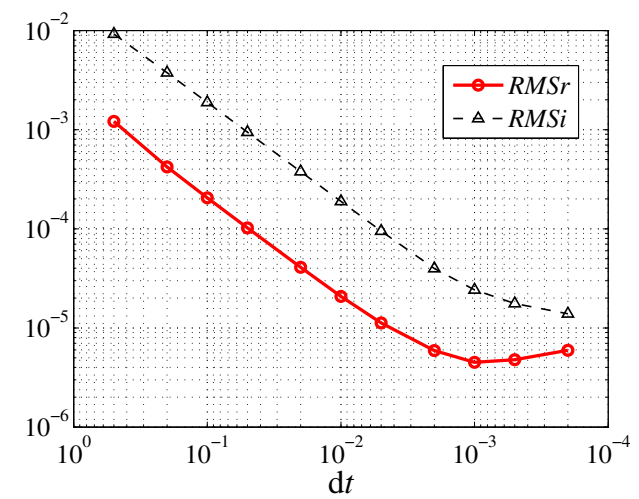

Figure 10: The RMS versus $d t$ obtained by the proposed scheme at $T=1(\mathrm{~s})$ for Example 3.2 .

$v=k_{1}^{2}+k_{2}^{2}-1$ and $\omega\left(x_{1}, x_{2}, t\right)=\left(k_{1}^{2}+k_{2}^{2}-1\right)\left(1-\cos ^{2} k_{1} x_{1} \cdot \cos ^{2} k_{2} x_{2}\right)$, where $k_{1}$ and $k_{2}$ are constants. The computational region with regular nodal distribution is as shown in Fig. 11, where $N=446$ containing 296 interior nodes and 150 boundary nodes. The exact solution is $u\left(x_{1}, x_{2}, t\right)=\cos \left(a x_{1}\right) \cos \left(b x_{2}\right) \exp (-i t)$. In the this example, $d t=0.005$ and $n s=10$ are used and the coefficients are assumed as $a=1, k_{1}=2$, and $k_{2}=3.5$.

In Fig. 12, the numerical solutions versus time (s) on the test line $x_{2}=0.586$ are reported. Compared with the analytical solutions, the absolute errors are shown in Fig. 13. From this figure, it can be seen that maximum absolute error is about $10^{-5}$ for both real and imaginary parts. Fig. 14 displays the RMSs of real and imaginary parts versus time (s) obtained by the LOOCV and the MST. From this figure, it can be found that although the minimum value of the RMS by the proposed MST is close to that of the LOOCV, most of the RMS obtained by MST is smaller than those by the LOOCV. It means the proposed MST can achieve better results. 


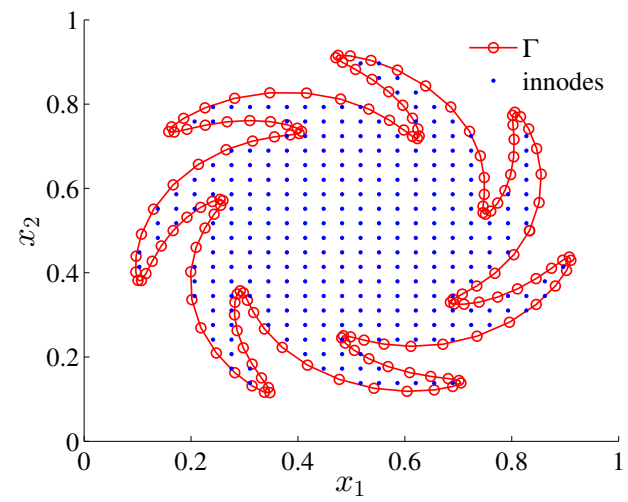

Figure 11: The closed boundary and collocation nodes for Example 3.3.
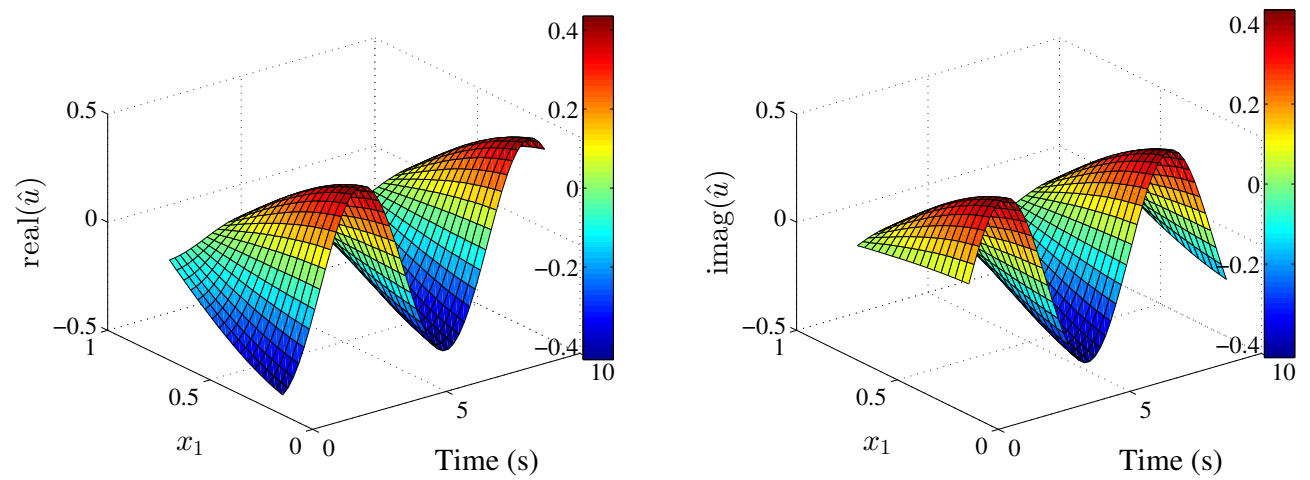

Figure 12: The real and imaginary parts of numerical solutions versus time $(\mathrm{s})$ on the test line $x_{2}=0.586$ for Example 3.3.
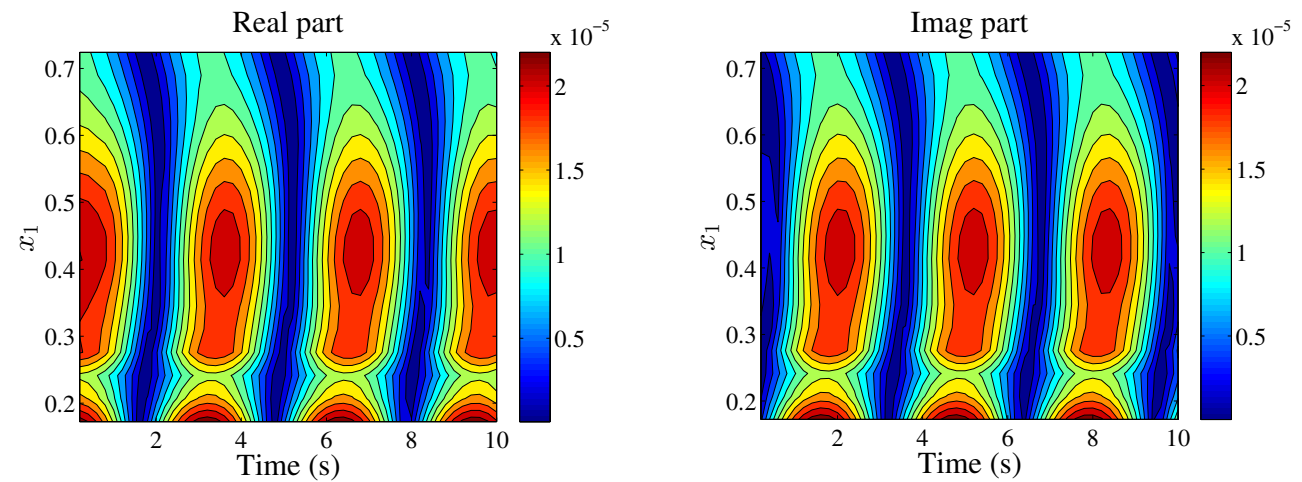

Figure 13: The obtained absolute error versus time (s) on the test line $x_{2}=0.586$ for Example 3.3. 

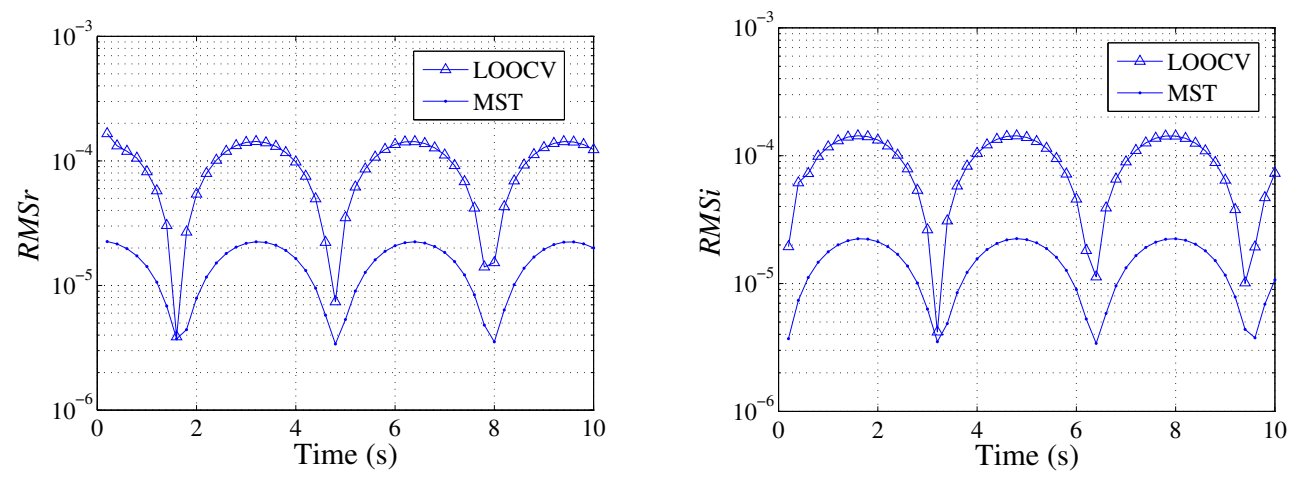

Figure 14: The comparison of the RMSs versus time (s) obtained by the LOOCV and the MST for Example 3.3.

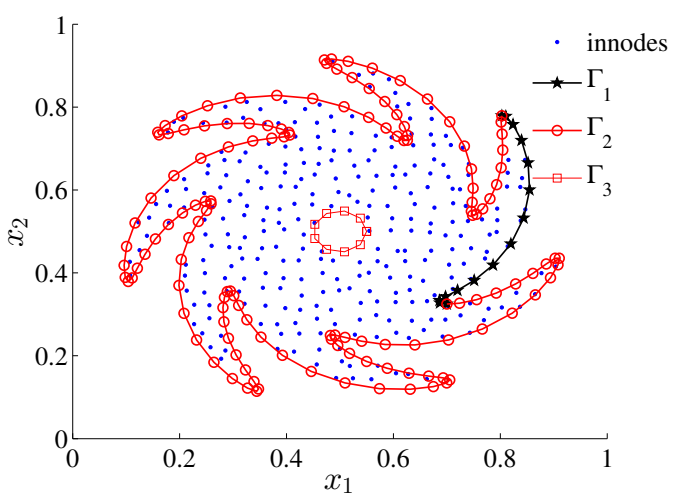

Figure 15: The closed boundary and collocation nodes for Example 3.4.

Example 3.4. In this example, we test a non-linear Schrödinger equation with $a=1$ and following potential functions

$$
\begin{aligned}
\omega\left(x_{1}, x_{2}, t\right)=\left(x_{1}^{2}-x_{2}^{2}\right)^{2} \exp (-2 t)+\left(1+x_{1} x_{2}\right)^{2} \\
\\
+\frac{i\left(x_{1}^{2}-x_{2}^{2}\right) \exp (-t)\left[\left(x_{1}^{2}-x_{2}^{2}\right) \exp (-t)-i\left(1+x_{1} x_{2}\right)\right]}{\left(x_{1}^{2}-x_{2}^{2}\right)^{2} \exp (-2 t)+\left(1+x_{1} x_{2}\right)^{2}},
\end{aligned}
$$

and

$$
v=-1
$$

The analytical solution is

$$
u\left(x_{1}, x_{2}, t\right)=\left(x_{1}^{2}-x_{2}^{2}\right) \exp (-t)+i\left(1+x_{1} x_{2}\right) .
$$

The numerical experiment is carried out on the region with randomly distributed nodes as shown in Fig. 15, where $\Gamma_{1}$ is the Neumann part of boundary, $\Gamma_{2}$ and $\Gamma_{3}$ denote the 

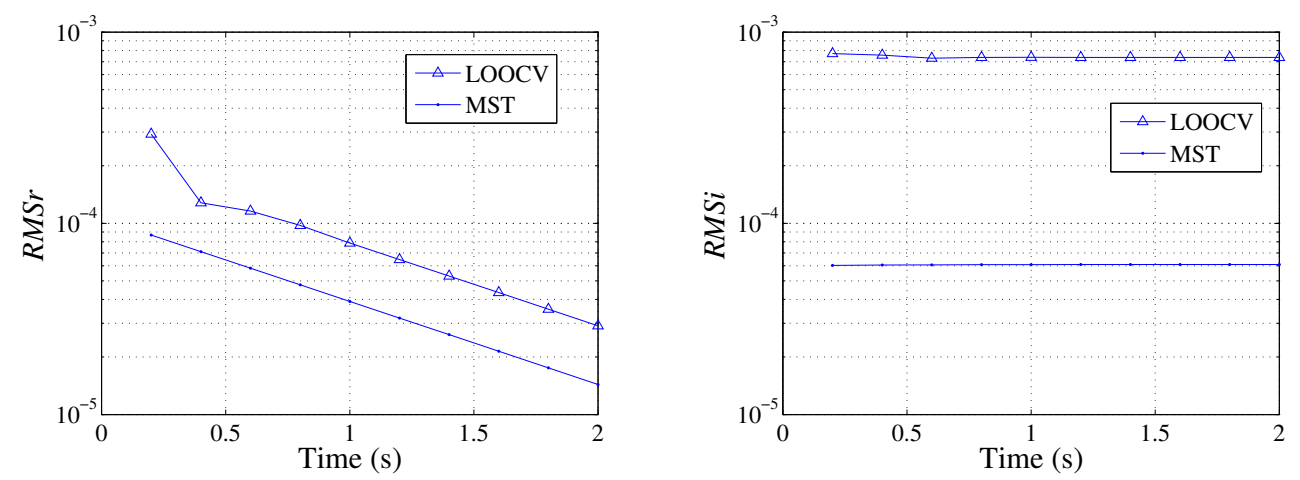

Figure 16: The real and imaginary parts of RMS versus time (s) for Example 3.4.
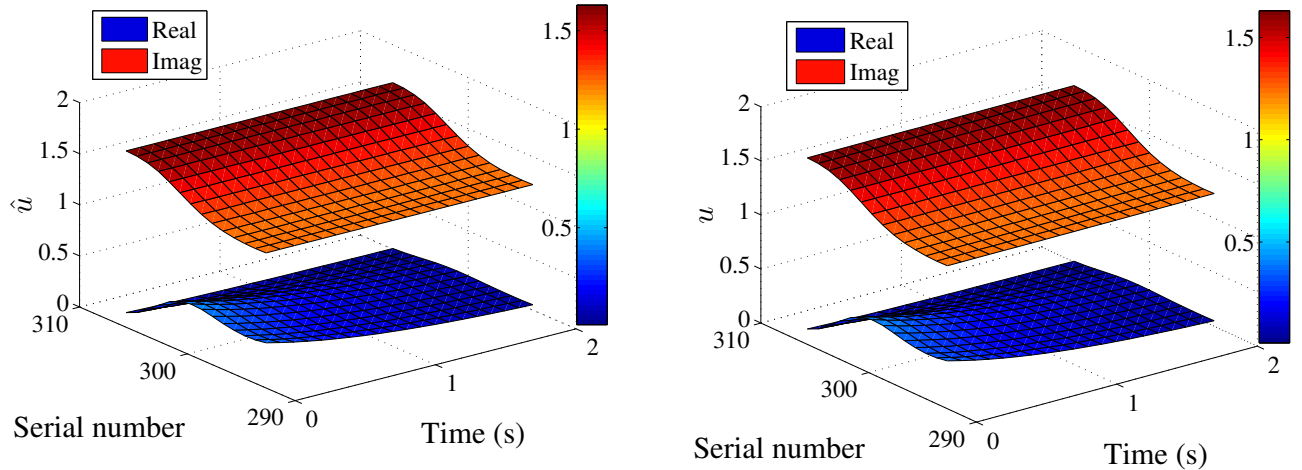

Figure 17: The comparison of numerical solutions and analytical solutions versus time (s) on Neumann boundary part $\Gamma_{1}$ for Example 3.4.

Direchlet parts of boundary, the total number of collocation nodes is $N=452$ containing 293 interior nodes, 14 boundary-nodes of Neumann part, and 136/9 boundary nodes of Direchlet parts $\Gamma_{2} / \Gamma_{3}$. In this example, $d t=0.005$ (s) and $n s=10$ are used.

Fig. 16 displays the real and imaginary parts of $R M S$ versus time (s) obtained by the proposed MST and the LOOCV. It can be seen that the proposed scheme achieves better results than the LOOCV. To further study the accuracy and stability of the proposed scheme, Fig. 17 presents the comparison of numerical solutions and analytical solutions and Fig. 18 shows the details about the relative error of $|\hat{u}|$ versus time (s) on the Neumann boundary part $\left(\Gamma_{1}\right)$. From these figures, the accuracy of the proposed scheme with small relative error less than $\left(10^{-3}\right)$ can be observed.

Example 3.5. In this example, the proposed scheme is compared with the approach described in [31] applied to a 3D non-linear Schrödinger equation on the bumpy sphere with regular distribution of collocation nodes, where $a=1$. The potential functions are as 


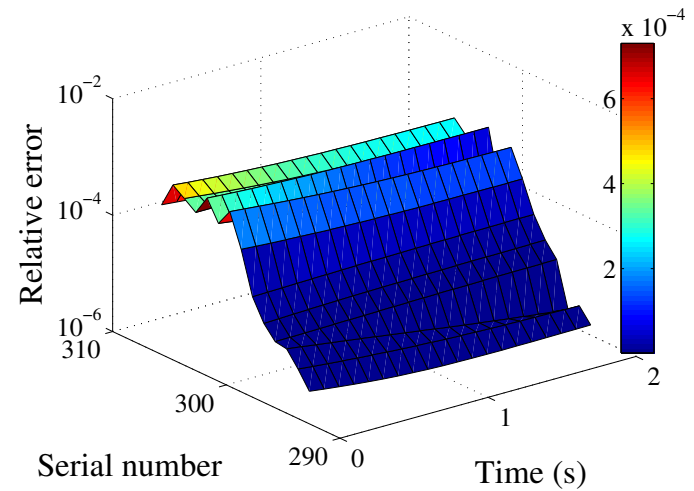

Figure 18: The relative error versus time (s) on Neumann boundary part $\Gamma_{1}$ for Example 3.4.

follows

$$
\omega\left(x_{1}, x_{2}, x_{3}, t\right)=\left(k_{1}^{2}+k_{2}^{2}+k_{3}^{2}-1\right)\left(1-\cos ^{2}\left(k_{1} x_{1}\right) \cos ^{2}\left(k_{2} x_{2}\right) \cos ^{2}\left(k_{3} x_{3}\right)\right),
$$

and

$$
v=k_{1}^{2}+k_{2}^{2}+k_{3}^{2}-1 .
$$

The analytical solution is given by [47]

$$
u\left(x_{1}, x_{2}, x_{3}, t\right)=\cos \left(k_{1} x_{1}\right) \cos \left(k_{2} x_{2}\right) \cos \left(k_{3} x_{3}\right) \exp (-i t) .
$$

Table 2 lists the comparison of RMS and elapsed time by the proposed MST and the LOOCV at different time history with $d t=0.01(\mathrm{~s}), n s=16, n b=1000$ and $n i=3108$. It can be seen that the results obtained by the proposed scheme are good as those by the LOOCV. It also shows that the proposed MST needs less computational time saving about 20 seconds compared to the LOOCV. To further study the accuracy and efficiency of the MST, Table 3 presents the comparison of the proposed MST and the LOOCV with different $n i / n b$ at $T=1$, where $d t=0.01, n s=16$. As listed in this table, we can find that the proposed MST achieves good results with less computational cost. It can be found that

Table 2: The comparison of RMS and elapsed time by the proposed MST and the LOOCV at different time history with $d t=0.01(\mathrm{~s}), n s=16, n b=1000$ and $n i=3108$ for Example 3.5.

\begin{tabular}{||c|c|c|c|c|c|c||}
\hline & \multicolumn{3}{|c|}{ MST } & \multicolumn{3}{c||}{ LOOCV } \\
\hline Time & RMSr & RMSi & Elapsed time & RMSr & RMSi & Elapsed time \\
\hline 0.5 & $6.50 e-5$ & $7.69 e-4$ & $6.15 e-0 \mathrm{~s}$ & $7.04 e-4$ & $4.46 e-4$ & $2.28 e+1 \mathrm{~s}$ \\
5 & $3.58 e-4$ & $8.97 e-5$ & $5.45 e+1 \mathrm{~s}$ & $2.36 e-4$ & $3.53 e-4$ & $7.24 e+1 \mathrm{~s}$ \\
10 & $7.49 e-5$ & $4.13 e-4$ & $1.08 e+2 \mathrm{~s}$ & $4.05 e-4$ & $2.02 e-4$ & $1.28 e+2 \mathrm{~s}$ \\
100 & $3.62 e-4$ & $2.29 e-4$ & $1.09 e+3 \mathrm{~s}$ & $1.13 e-4$ & $4.43 e-4$ & $1.12 e+3 \mathrm{~s}$ \\
\hline
\end{tabular}


Table 3: The comparison of the proposed MST and the LOOCV with different $n i / n b$ at $T=1$, where $d t=0.01$ (s), $n s=16$ for Example 3.5.

\begin{tabular}{||c|c|c|c|c|c|c||}
\hline & \multicolumn{3}{|c|}{ MST } & \multicolumn{3}{c||}{ LOOCV } \\
\hline$n i / n b$ & $R M S r$ & $R M S i$ & Elapsed time & $R M S r$ & $R M S i$ & Elapsed time \\
\hline $748 / 500$ & $2.16 e-4$ & $2.43 e-4$ & $1.95 e-0 \mathrm{~s}$ & $2.90 e-4$ & $5.23 e-4$ & $5.49 e-0 \mathrm{~s}$ \\
$3108 / 1000$ & $8.80 e-5$ & $1.30 e-5$ & $1.44 e+1 \mathrm{~s}$ & $1.54 e-4$ & $1.52 e-4$ & $2.85 e+1 \mathrm{~s}$ \\
$5309 / 3800$ & $5.07 e-5$ & $1.47 e-4$ & $2.61 e+1 \mathrm{~s}$ & $1.24 e-4$ & $4.33 e-5$ & $5.89 e+1 \mathrm{~s}$ \\
\hline
\end{tabular}

the more collocation nodes are used, the more cost by the MST are saved. Figs. 19 and 20 present the RMS and elapsed time versus $n s$ by the proposed MST and the LOOCV with $d t=0.01(\mathrm{~s}), n b=1000$, and $n i=3108$. These results further demonstrate that the propose MST is accurate.
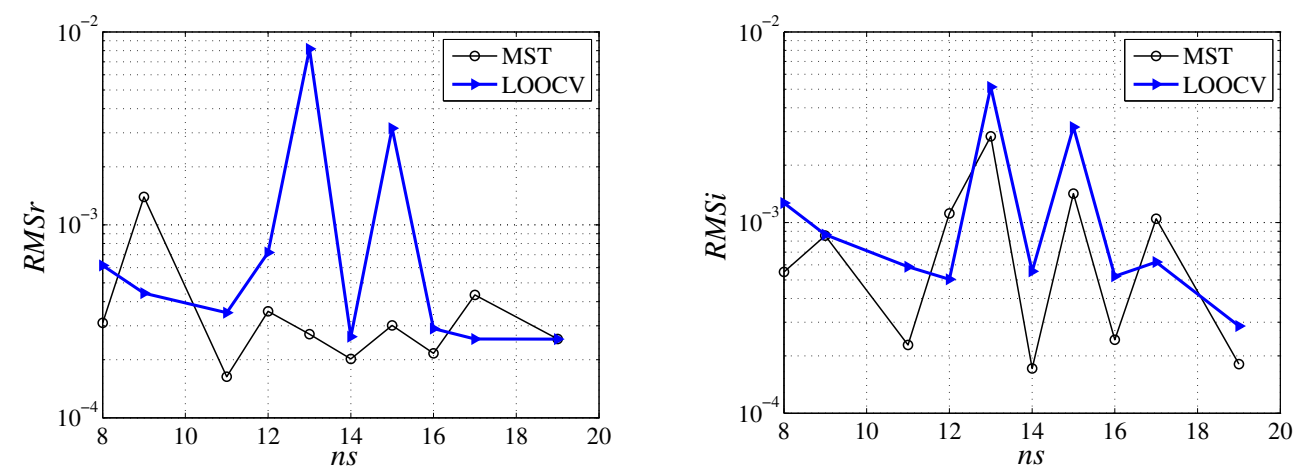

Figure 19: The real and imaginary parts of RMS versus ns by the proposed MST and the LOOCV with $d t=0.01(\mathrm{~s}), n b=1000$, and $n i=3108$ for Example 3.5.

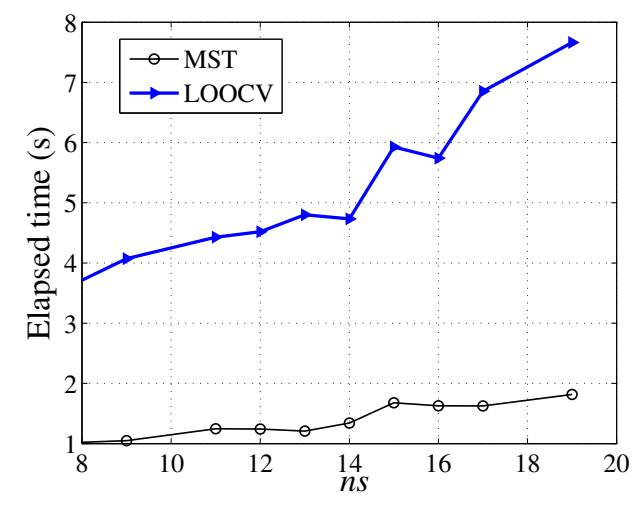

Figure 20: The comparison of the elapsed time versus $n s$ by the MST and the LOOCV for Example 3.5. 
Example 3.6. In last example, the proposed scheme is further compared with that in [31] where a 3D non-linear Schrödinger equation on the bumpy sphere with regular distribution of collocation nodes with $a=0.5$ is considered. The potential functions are as follows

$$
\omega\left(x_{1}, x_{2}, x_{3}, t\right)=\frac{3}{4\left(1-3 K^{2}\right)} \operatorname{sech}^{2}\left(\frac{x_{1}+x_{2}+x_{3}-4 K t}{2 \sqrt{3-9 K^{2}}}\right)
$$

and

$$
v=0 .
$$

The analytical solution [48] is as follows

$$
u\left(x_{1}, x_{2}, x_{3}, t\right)=\frac{3 \sqrt{2}}{4 \sqrt{1-3 K^{2}}} \operatorname{sech}^{2}\left(\frac{x_{1}+x_{2}+x_{3}-3 K t}{2 \sqrt{3-9 K^{2}}}\right) e^{\left(\mathrm{i} K\left(x_{1}+x_{2}+x_{3}+\frac{t}{2 K} \frac{1-3 K^{2}+9 K^{4}}{1-3 K^{2}}\right)\right)} .
$$

Figs. 21 and 22 display the RMS and the elapsed time versus time (s) by the proposed MST and the LOOCV with $d t=0.1(\mathrm{~s}), d t=0.01(\mathrm{~s}), n b=500$, and $n i=748$. From Fig. 21 , it can be observed that both of the proposed scheme and the LOOCV achieve good results with high accuracy $\left(10^{-4}\right)$ with $d t=0.01$. Good convergency of both of them can also be found. In Fig. 22, we can find the proposed MST needs less computational time than the LOOCV saving about 5 seconds. In details, Figs. 23 and 24 focus on a test node $(-0.4412$, $0.4412,-0.0882)$. The comparison of the analytical and numerical solutions on this test node is as shown in Fig. 23 and the absolute error on the node is presented by Fig. 24. From these figures, we can find that the obtained numerical solutions by both of the MST and the LOOCV are quite consistent with the analytical solutions with small error below $10^{-3}$. It demonstrates that the proposed MST is accurate and stable.
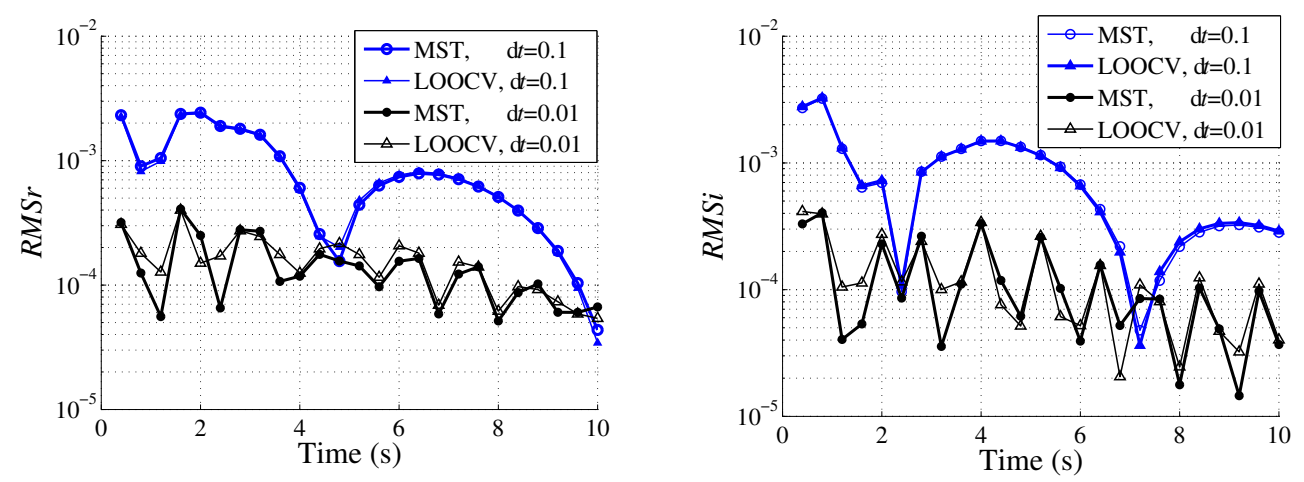

Figure 21: The real and imaginary parts of RMS versus time (s) by the proposed MST and the LOOCV with $d t=0.1(\mathrm{~s}), d t=0.01(\mathrm{~s}), n b=500$, and $n i=748$ for Example 3.6. 


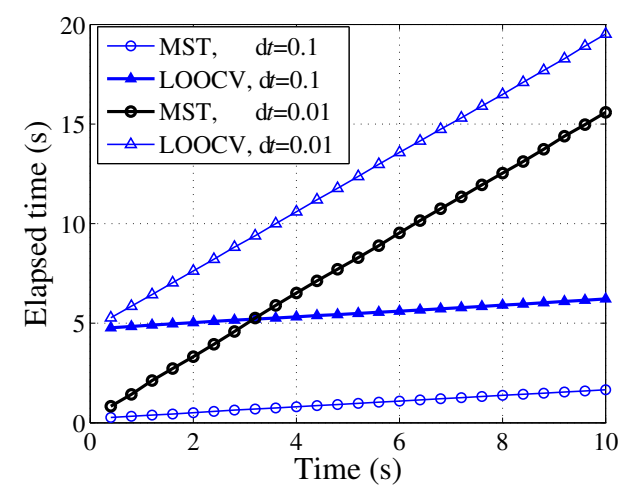

Figure 22: The comparison of the elapsed time versus time (s) by the proposed MST and the LOOCV with $d t=0.1(\mathrm{~s}), d t=0.01(\mathrm{~s}), n b=500$, and $n i=748$ for Example 3.6.

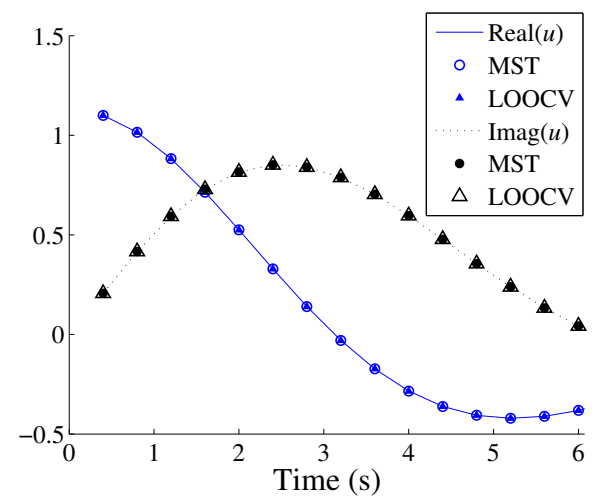

Figure 23: The comparison of the analytical and numerical solutions on a test node by the proposed MST and the LOOCV with $d t=0.01(\mathrm{~s}), n b=500$, and $n i=748$ for Example 3.6.

\section{Conclusions}

In this paper, we apply the localized method of approximate particular solutions (LMAPS) for 2D and 3D non-linear Schrödinger equations in irregular domain with complex boundary conditions. In the proposed scheme, the multiple-scale technique (MST) is applied in determination of the shape parameters of MQ-RBF and extended to determination of the shape parameters of Gaussian function for 3D problems. The matrices obtained by the localized scheme can be a sparse system, which can save computational cost. Examples are designed to validate the effectiveness of the proposed scheme with regular or irregular distribution of collocation nodes. Compared with the LMAPS using the $c_{\text {optimal }}$ and the LOOCV for determination of shape parameters, numerical results demonstrate the accuracy and efficiency of the proposed MST. The obtained results also show that the proposed scheme preserves good stability and efficiency for the 


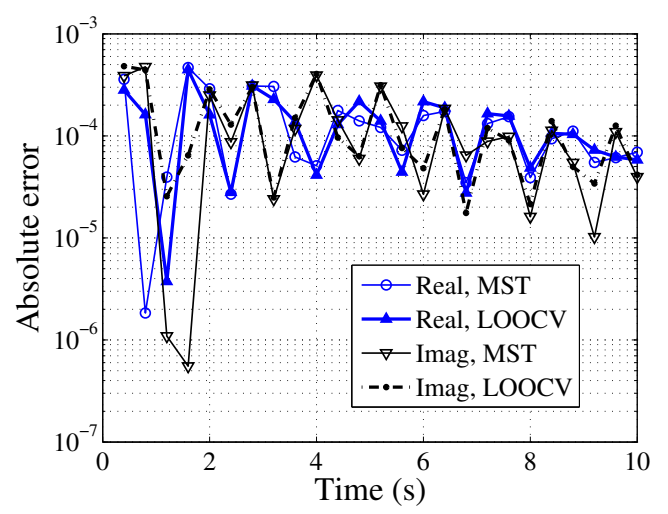

Figure 24: The absolute error on a test node by the proposed MST and the LOOCV with $d t=0.01(\mathrm{~s}), n b=500$, and $n i=748$ for Example 3.6.

Schrödinger equations in arbitrary domain with complex boundary conditions and the MST has good potential in solving three-dimensional problems.

\section{Acknowledgements}

The authors thank the editor and anonymous reviewers for their constructive comments on the manuscript. The research of the authors was supported by the Natural Science Foundation of Jiangsu Province (No. BK20150795), the Fundamental Research Funds for the Central Universities (No. 2018B16714), the National Natural Science Foundation of China (Nos. 11702083, 51679150, 51579153, 51739008, 51527811), the State Key Laboratory of Mechanics and Control of Mechanical Structures (Nanjing University of Aeronautics and Astronautics) (No. MCMS-0218G01), the National Key R\&D Program of China (No. 2016YFC0401902), and the Fund Project of NHRI (Nos. Y417002, Y417015).

\section{References}

[1] V. KorePIN AND N. SLAVNOV, Time and temperature dependent correlation functions of $1 d$ models of quantum statistical mechanics, Phys. Lett. A, 236 (1997), pp. 201-205.

[2] H. D. ZhANG, R. X. XU, X. Zheng AND Y. J. YAN, Statistical quasi-particle theory for open quantum systems, Mol. Phys., 116 (2018), pp. 1-33.

[3] A. R. Osborne, M. OnORAto AND M. Serio, The nonlinear dynamics of rogue waves and holes in deep-water gravity wave train, Phys. Lett. A, 275 (2000), pp. 386-393.

[4] M. Onorato, S. Residori, U. Bortolozzo, A. Montina And F. T. Arecchi, Rogue waves and their generating mechanisms in different physical contexts, Phys. Rep., 528 (2013), pp. 47-89.

[5] E. M. Bitner-Gregersen, S. K. Bhattacharya, I. K. Chatjigeorgiou, I. Eames, K. ELLERMANN AND K. EWANS ET AL., Recent developments of ocean environmental description with focus on uncertainties, Ocean Eng., 86 (2014), pp. 26-46. 
[6] S. E. Hirdaris, W.Bai, D. Dessi, A. Ergin, X. Gu and O. A. Hermundstad et al., Loads for use in the design of ships and offshore structures, Ocean Eng., 78 (2014), pp. 131-174.

[7] Z. H. GAN, B. L. GUO AND X. JIANG, Orbital instability of standing waves for the generalized 3d nonlocal nonlinear Schrödinger equations, Acta Math. Sci., 35 (2015), pp. 1163-1188.

[8] G. LI, S. PENG AND C. WANG, Infinitely many solutions for nonlinear Schrödinger equations with electromagnetic fields, J. Differ. Equ., 251 (2011), pp. 3500-3521.

[9] K. X. GuO, T. P. DAS AND C. Y. CHEN, Studies on the electro-optic effects of doublelayered quantum wires in magnetic fields, Phys. B Condensed Matter, 293 (2000), pp. 11-15.

[10] A. Biswas, A. Moran, D. Milovic, F. Majid AND K. C. Biswas, An exact solution for the modified nonlinear Schrödinger's equation for davydov solitons in alpha-helix proteins, Math. Biosci., 227 (2010), pp. 68-71.

[11] M. J. D. RAMSTEAD, P. B. BADCOCK AND K. J. FRISTON, Answering Schrödinger's question: A free-energy formulation, Phys. Life Rev., 24 (2017), pp. 1-16.

[12] Y. GU, L. WANG AND W. CHEN ET AL., Application of the meshless generalized finite difference method to inverse heat source problems, Int. J. Heat Mass Transf., 108 (2017), pp. 721-729.

[13] Y. SuN, Indirect boundary integral equation method for the Cauchy problem of the Laplace equation, J. Sci. Comput., 71 (2017), pp. 469-498.

[14] B. WANG AND D. LIANG, The finite difference scheme for nonlinear Schrödinger equations on unbounded domain by artificial boundary conditions, Appl. Numer. Math., 128 (2018), pp. 183204.

[15] X. ZHU, Z. YUAN AND J. WANG ET AL., Finite element method for time-space-fractional Schrodinger equation, Electron. J. Differ. Equ., 166 (2017), pp. 1-18.

[16] Y. GU, X. HE AND W. CHEN ET AL., Analysis of three-dimensional anisotropic heat conduction problems on thin domains using an advanced boundary element method, Comput. Math. Appl., 75 (2018), pp. 33-44.

[17] X. ZhaO, Z. Z. Sun AND H. Z. PENG, A fourth-order compact ADI scheme for 2D nonlinear space fractional Schrödinger equation, SIAM J. Sci. Comput., 36 (2014), pp. A2865-A2886.

[18] A. H. BHRAWY AND M. A. ZAKY, An improved collocation method for multi-dimensional spacetime variable-order fractional Schrödinger equations, Appl. Numer. Math., 111 (2017), pp. 197218.

[19] L. W. ZHANG AND K. M. LiEW, An element-free based solution for nonlinear Schrödinger equations using the ICVMLS-Ritz method, Appl. Math. Comput., 249 (2014), pp. 333-345.

[20] L. W. ZHANG, Y. J. DENG AND K. M. LIEW ET AL., The improved complex variable element-free Galerkin method for two-dimensional Schröodinger equation, Comput. Math. Appl., 68 (2014), pp. 1093-1106.

[21] B. F. N. FLYER, Solving PDEs with radial basis functions, Acta Numer., 24 (2015), pp. 215-258.

[22] W. Z. QU AND W. CHEN, Solution of two-dimensional stokes flow problems using improved singular boundary method, Adv. Appl. Math. Mech., 7 (2015), pp. 13-30.

[23] J. P. LI AND W. CHEN, A modified singular boundary method for three-dimensional high frequency acoustic wave problems, Appl. Math. Model., 54 (2018), pp. 189-201

[24] Y. HONG, J. LIN AND W. CHEN, A typical backward substitution method for the simulation of Helmholtz problems in arbitrary 2D domains, Eng. Ana. Bound. Elem., 93 (2018), pp. 167-176.

[25] F. J. WANG, Q. S. HUA AND C. S. LIU, Boundary function method for inverse geometry problem in two-dimensional anisotropic heat conduction equation, Appl. Math. Lett., 84 (2018), pp. 130136.

[26] J. LIN, C. S. CHEN, C. S. LIU AND J. LU, Fast simulation of multi-dimensional wave problems by the sparse scheme of the method of fundamental solutions, Comput. Math. Appl., 72 (2016), pp. 
$555-567$.

[27] J. LIN, C. Z. ZHANG, L. L. SUN AND J. LU, Simulation of seismic wave scattering by embedded cavities in an elastic half-plane using the novel singular boundary method, Adv. Appl. Math. Mech., 10 (2018), pp. 322-342.

[28] J. LIN, W. CHEN AND F. Z. WANG, A new investigation into regularization techniques for the method of fundamental solutions, Math. Comput. Simulat., 81 (2011), pp. 1144-1152.

[29] M. DEHGHAN, M. ABBASZADEH AND A. MOHEBBI, The numerical solution of nonlinear high dimensional generalized Benjamin-Bona-Mahony-Burgers equation via the meshless method of radial basis functions, Comput. Math. Appl., 68 (2014), pp. 212-237.

[30] B. Fornberg, E. LARSSON AND N. Flyer, Stable Computations with Gaussian Radial Basis Functions in 2-D, Siam J. Sci. Comput., 33 (2011), pp. 869-892.

[31] J. LIN, Y. HONG AND L. H. KUO ET AL., Numerical simulation of 3D nonlinear Schrödinger equations by using the localized method of approximate particular solutions, Eng. Anal. Bound. Elem., 78 (2017), pp. 20-25.

[32] J. Lin, C. S. CHEN, F. J. WANG AND T. DANGAL, Method of particular solutions using polynomial basis functions for the simulation of plate bending vibration problems, Appl. Math. Model., 49 (2017), pp. 452-469.

[33] C. C. TSAI, Z. H. LIN AND T. W. HsU, Using a local radial basis function collocation method to approximate radiation boundary conditions, Ocean Eng., 105 (2015), pp. 231-241.

[34] M. DEHGHAN AND A. NiKPOUR, Numerical solution of the system of second-order boundary value problems using the local radial basis functions based differential quadrature collocation method, Appl. Math. Model., 37 (2013), pp. 8578-8599.

[35] G. YAO, J. KOLIBAL AND C. S. CHEN, A localized approach for the method of approximate particular solutions, Comput. Math. Appl., 61 (2011), pp. 2376-2387.

[36] W. CHEN, Y. HONG AND J. LIN, The sample solution approach for determination of the optimal shape parameter in the Multiquadric function of the Kansa method, Comput. Math. Appl., 75 (2018), pp. 2942-2954.

[37] C. S. LIU, W. CHEN AND Z. J. FU, A multiple-scale MQ-RBF for solving the inverse Cauchy problems in arbitrary plane domain, Eng. Anal. Bound. Elem., 68 (2016), pp. 11-16.

[38] J. L. Bentley, Multidimensional binary search trees used for associative searching, Commun. Acm., 18 (1975), pp. 509-517.

[39] G. YAO, An improved localized method of approximate particular solutions for solving elliptic PDEs, Comput. Math. Appl., 71 (2016), pp. 171-184.

[40] X. ZHANG, M. CHEN AND C. S. CHEN ET AL., Localized method of approximate particular solutions for solving unsteady Navier-Stokes problem, Appl. Math. Model., 40 (2016), pp. 22652273.

[41] J. SU, F. ZHU, Y. GENG AND X. Ni, Numerical study of wave overtopping based on local method of approximate particular solution method, Adv. Mech. Eng., 6 (2014), pp. 541717.

[42] C. M. FAN, C. H. YANG AND W. S. LAI, Numerical solutions of two-dimensional flow fields by using the localized method of approximate particular solutions, Eng. Anal. Bound. Elem., 57 (2015), pp. 47-57.

[43] J. Lin, W. CHEN AND K. Y. SzE, A new radial basis function for Helmholtz problems, Eng. Anal. Bound. Elem., 36 (2012), pp. 1923-1930.

[44] J. LIN, S. Y. REUTSKIY AND J. LU, A novel meshless method for fully nonlinear advectiondiffusion-reaction problems to model transfer in anisotropic media, Appl. Math. Comput., Accepted.

[45] Y. XU AND C. W. SHU, Local discontinuous galerkin methods for nonlinear Schröodinger equations, 
J. Comput. Phys., 205 (2005), pp. 72-97.

[46] A. MOHEBBI AND M. DEHGHAN, The use of compact boundary value method for the solution of two-dimensional Schrödinger equation, J. Comput. Appl. Math., 225 (2009), pp. 124-134.

[47] G. YAO AND Z. YU, A localized meshless approach for modeling spatial-temporal calcium dynamics in ventricular myocytes, Int. J. Num. Meth. Bio. Eng., 28 (2012), pp. 187-204.

[48] J. XIA, S. HAN AND M. WANG, The exact solitary wave solutions for the Kleingordon-Schrödinger equations, Appl. Math. Mech., 23 (2002), pp. 58-64. 